\title{
3 combinations of modifiable lifestyle behaviours reduced the risk of coronary artery disease
}

Stampfer MJ, Hu FB, Manson JE, et al. Primary prevention of coronary heart disease in women through diet and lifestyle. N Engl J Med 2000 Jul 6;343:16-22.

\section{QUESTION: Which combinations of lifestyle behaviours are associated with the reduced risk of coronary artery disease (CAD)?}

Source of funding: US National Institutes of Health.

For correspondence: Dr M J Stampfer, Department of Epidemiology, Harvard School of Public Health 677 Huntington Avenue, Boston, MA 02115 .

USA.Fax +1617566 7805.

\section{Setting}

USA.

\section{Participants}

84129 women who were 34-59 years of age in 1980 (baseline). Exclusion criteria were $\geqslant 10$ non-responses to dietary questions; implausible scores for total food intake; and previous cancer, diabetes, angina, myocardial infarction (MI), stroke, or other cardiovascular disease.

\section{Assessment of risk factors}

Nurses completed postal questionnaires that collected information on age, height and weight, current and past smoking, parental history of MI, menopausal status including the use of postmenopausal hormone replacement therapy, diagnosis of hypertension and high cholesterol concentrations, physical activity, and dietary intake. Follow up questionnaires were completed every 2 years. Low risk was defined as not currently smoking, a body mass index $(\mathrm{BMI})<25$, mean intake of $\geqslant 5 \mathrm{~g}$ of alcohol/day, mean of $\geqslant 0.5$ hours/day of moderate or vigorous physical activity, and a score in the highest $40 \%$ of the cohort based on a composite measure of diet (low in trans fat and glycaemic load; high in cereal fibre, marine n-3 fatty acids, and folate; and a high ratio of polyunsaturated to saturated fat). For analysis, combinations of 3 low risk factors (diet, smoking, and exercise), 4 low risk factors (diet, smoking, exercise, and BMI), and 5 low risk factors (diet, smoking, exercise, BMI, and alcohol use) were used.

\section{Main outcome measures}

Death, non-fatal MI, and stroke.

\section{Main results}

During follow up, 1128 major coronary events (296 deaths from CAD and 832 non-fatal MIs) and 705 strokes were reported. Each of the 5 low risk factors independently predicted the risk of CAD or stroke. The risks of CAD and $\mathrm{CAD}$ or stroke were reduced by having combinations of 3,4 , and 5 low risk factors (table).

\section{Conclusion}

3 combinations of modifiable lifestyle behaviours (diet, smoking, exercise, body mass index, and alcohol use) reduced the risk of major coronary artery disease events and stroke.

Multivariate adjusted relative risks (RRs) for coronary artery disease (CAD) and CAD or stroke in women at 14 years of follow up*

\begin{tabular}{|c|c|c|}
\hline Combination of low risk factors & $\begin{array}{l}\text { RR }(95 \% \mathrm{Cl}) \text { for } \\
\text { CAD }\end{array}$ & $\begin{array}{l}\text { RR (CI) for CAD or } \\
\text { stroke }\end{array}$ \\
\hline Diet, smoking, and exercise $†$ & $0.43(0.33$ to 0.55$)$ & $0.46(0.37$ to 0.55$)$ \\
\hline $\begin{array}{l}\text { Diet, smoking, exercise, and body mass } \\
\text { index } \neq\end{array}$ & 0.34 (0.23 to 0.52$)$ & $0.38(0.28$ to 0.51$)$ \\
\hline $\begin{array}{l}\text { Diet, smoking, exercise, body mass index, } \\
\text { and alcohol use }\end{array}$ & $0.17(0.07$ to 0.41$)$ & $0.25(0.14$ to 0.44$)$ \\
\hline
\end{tabular}

\section{COMMENTARY}

The results of the study by Stampfer $e t$ al add to the expanding evidence base that a healthy lifestyle through diet, not smoking, and regular exercise can decrease the risk of developing $\mathrm{CAD}$. The findings also suggest that additional risk reduction may be obtained by maintaining a BMI $<25$ and through moderate use of alcohol. This study did not evaluate endpoints associated with the negative effects of alcohol use or identify an upper limit for alcohol consumption, so caution should be used before recommending alcohol use to patients. Furthermore, the study controlled for, but did not consider, the additional effects of pharmacological treatment and other concurrent medical conditions.

The results are clinically relevant to nurses working with adult clients and are personally relevant to individual nurses. In this study population, only about $3 \%$ of nurses met the criteria for adherence to a low risk lifestyle despite their role as health educators. This suggests that although nurses are likely to be knowledgeable about the risk factors for CAD, they still may have problems adopting the behaviours that constitute a low risk lifestyle.

These findings indicate that $82 \%$ of coronary events might have been prevented if all women were in the low risk group. Many women might not be able to make the substantial changes required to be classified as having a low risk lifestyle. Women should be told, however, that a gradient of risk was present within the categories of each behaviour, which suggests that even small changes in lifestyle can have an important effect in the prevention of CAD. When interacting with patients, nurses now have further evidence that initial modifications should be made in diet, smoking, and exercise as these are the 3 most powerful modifiable risk factors. Clearly, there is more research to be done around how to support and enhance behaviour change related to lifestyle modification.

Diane Wehby, RN, CCRN, MSN Director of Critical Care and Cardiovascular Services Detroit Medical Center-Sinai-Grace Hospital Detroit, Michigan, USA 